\title{
Novice Occupational Therapist's Experience of Working in Neonatal Intensive Care Units in KwaZulu-Natal
}

\author{
*Michaela Hardy; B.OT (UKZN), M.OT (UKZN). https://orcid.org/0000-000 I-8928-6 I 37 \\ Occupational Therapist at Linden Lodge School, 61 Princes Way, London, United Kingdom.
}

Pragashnie Govender; B.OT (UDW), M.OT (UKZN), PhD (UKZN). https://orcid.org/0000-0003-3 I55-3743

Associate Professor at UKZN, Occupational Therapy, School of Health Sciences, University of KwaZulu Natal (Westville Campus), South Africa.

Deshini Naidoo; B.OT (UDW), M.OT (UKZN), PhD (UKZN). https://orcid.org/0000-000 I-6276-22 IX Academic Leader at UKZN, Discipline of Occupational Therapy, School of Health Sciences University of KwaZulu Natal (Westville Campus), South Africa.

Introduction: The neonatal intensive care unit, an environment designed to meet the needs of severely ill neonates, is an area of practice for occupational therapists. However, there is limited evidence available around the training and practice of South African occupational therapists in these units.

Aim: To explore community service occupational therapists' experiences of working in neonatal intensive care units in the KwaZuluNatal public health sector.

Methodology: This study followed an explorative qualitative design. Homogenous purposive sampling was employed to recruit 12 therapists that participated in in-depth interviews. Interviews were audio-recorded and transcribed. Data were analysed thematically by inductive reasoning initially, followed by categorisation via deductive reasoning using the theory of occupational adaptation.

Results: Three themes emerged; the desire for mastery (including intrinsic drivers, multiple roles and routines in the NICU and capabilities and prior experiences); demand for mastery (including barriers and enablers in the NICU environment and development of the therapist-client relationship) and press for mastery (development of occupational identity, competence and adaptation).

Conclusions: The newly qualified occupational therapists who participated in this study appeared to be able to overcome the challenges of working in the highly technical environment of the NICU. There is a need for greater support and training of community service occupational therapists in this specialised field of practice.

Key words: occupational therapy training, community service occupational therapists, neonatal intensive care units, clinical experiences, public health sector

\section{INTRODUCTION}

The neonatal period is a vulnerable phase posing many health risks. Preventing deaths during this period is a major global concern'. In a middle-income country such as South Africa, spontaneous pre-term births and birth trauma are leading causes of death'. The World Health Organisation (WHO) established 'goal three' of the Sustainable Development Goals (SDG), which aims to reduce neonatal deaths to 12 per 1000 live births and eliminate all preventable deaths in children under the age of five by the year 2030'. Accordingly, the South African Department of Health (DOH) has aligned their vision of reducing maternal and child mortality as a priority in the National Service Delivery Agreement ${ }^{3}$.

The neonatal intensive care unit (NICU) is a specialised environment designed to meet the needs of severely ill neonates ${ }^{4}$. The Nurturing Care Framework, endorsed by WHO, promotes a holistic view of care by focusing on infant health, nutrition, competent caregivers, security and safety, as well as early learning opportunities'. Given this holistic view, the occupational therapist has an important role to play, as part of the multidisciplinary team (MDT), within the NICU5. However, this may be daunting for new therapists delivering services in this high-risk environment ${ }^{6,7}$, as the services provided by occupational therapists require specific knowledge, skills, and equipment to ensure neonate safety ${ }^{4}$. Globally, the role of the occupational therapists in a NICU is well defined, and the occupational therapist is viewed as a valuable member of the MDT, performing functions such as splinting, positioning and handling, feeding, providing caregiver support, and implementing environmental alterations ${ }^{7}$. However, many occupational therapists may feel incompetent in delivering specialised NICU services due to lack of training or not having prior experience ${ }^{8}$.

Community service in South Africa is a compulsory year of supervised practice for health professionals, after a four-year occupational therapy undergraduate degree, followed by registration as an independent occupational therapy practitioner with the Health Professions Council of South Africa (HPCSA). Community service occupational therapists (CSOTs) have raised issues related to their 
lack of preparedness for delivering specialised services in NICUs due to their limited exposure during undergraduate training ${ }^{9}$. Despite this limited training, newly qualified occupational therapists in South Africa are still required to assume roles in high-care settings such as the NICU within the public sector ${ }^{7}$. In the South African context, there is limited literature about the role and scope of occupational therapists in the NICU. Additionally, there is limited literature that explores how community service therapists adapt to the demands of working in NICUs in public sector hospitals. This study therefore aimed at gaining an understanding of occupational therapist CSOTs' experiences of working in NICUs in the KwaZulu-Natal (KZN) public health sector. It is important to understand the process of adaptation the CSOTs undergo, as this aids in the development of their identity and the role they play within NICUs, which ultimately influences service delivery in this area.

\section{LITERATURE REVIEW}

The NICU is a setting that caters for high-risk neonates and new-born infants who demonstrate signs of the increased possibility of morbidity or mortality, focusing on family-based care $^{4}$. NICU's require adequate facilities and highly trained professionals to deal with the infants' specific needs ${ }^{4}$. Globally, working in a NICU is recognised as an advanced area of practice, and health professionals are required to have advanced clinical reasoning skills and specialist abilities to deal with this technical and emotionally taxing field of work ${ }^{10}$. Every year, approximately four million neonates die globally, with most deaths occurring within the first 24 hours of life" of death of neonates worldwide include infections, pre-term births and asphyxia, with low birth weight (LBW) being a serious risk factor for further complications ${ }^{\prime \prime}$. Modern advances in neurodevelopment have led to a shift towards focusing more on the future developmental outcomes of the infant ${ }^{12}$. This has resulted in increased research into how the NICU environment, parental stress and healthcare providers influence the infant's neurodevelopment; with the importance of the parental role being recognised worldwide and increased efforts have been made to encourage family participation ${ }^{7,8,11}$.

Most neonatal deaths in South Africa are caused by asphyxia, prematurity, LBW and infections, which parallels global statistics ${ }^{2,13}$. A retrospective study at a rural hospital in the KZN province, which focused on monitoring the neonatal admissions, deaths, and discharges in the NICU, found that a very low birth weight (VLBW) and prematurity were high predictors of death, usually occurring within the first three days following admission ${ }^{2}$. The authors of this study concluded that many of these deaths could be avoidable with a better quality of care ${ }^{2}$. Similarly, a study that accessed participants in the central and eastern regions of Tshwane, found many preventable factors associated with neonatal mortality including poor accessibility to NICU beds with ventilators, hospital-acquired infections, delayed seeking of medical help and inadequately trained healthprofessionals $^{14}$. Despite of the mentioned South African research, early intervention is often not initiated in diverse contexts due to inaccessible resources, poverty factors and a lack of help-seeking behaviour because of low education levels and services not made well known to the public ${ }^{13}$. NICU facilities are required at all regional and tertiary hospitals in South Africa. However, most births occur in district hospitals or community health centres; therefore, it is crucial that neonatal services are available at these levels with a high care facility ${ }^{15}$. Occupational therapists working in district hospitals, mainly community service therapists, may be required to deliver services to this vulnerable population ${ }^{16}$.

Occupational therapists working in the NICUs in KwaZuluNatal are often faced with the burden of increasingly large numbers of patients due to the high birth rate in this province ${ }^{15}$. Additionally, most persons living in $\operatorname{KZN}(89,9 \%)$ have a low to middle socioeconomic status, with high poverty and unemployment rates ${ }^{15}$. Furthermore, KwaZulu-Natal also has the highest number of social grant beneficiaries out of all nine provinces in South Africa $(23,2 \%)$, with high numbers of Care Dependency and Child Support Grant beneficiaries ${ }^{15}$. This means that the CSOT may also have to consider severe financial restrictions and supplying low-cost interventions to the families in the NICU. The South African context is unique and therefore the effect of the environment is required to understand service delivery. Culture is an important aspect to consider especially when providing maternal and child healthcare ${ }^{17}$. Many cultural practices, especially regarding mother and infant care, continue despite limited knowledge of potential health benefits or possible harm, specifically in developing countries. This is usually due to family pressure, set routines or convenience ${ }^{17}$.

Many neonatal deaths are preventable with good quality of care; therefore, to reach the SDGs, certain standards need to be established and achieved. Lincare ${ }^{18}$ postulated that specific standards should be in place to provide quality child healthcare. This includes referrals to advanced or specialist care units for complex cases and an appropriate number of nursing staff working in NICUs ${ }^{18}$. It was suggested that all staff working in the NICU should have a thorough introduction and training on arrival as well as periodic in-services to ensure continual professional development in this setting ${ }^{18}$. Additionally, the NICU's physical environment requires careful consideration such as ensuring adequate lighting, ventilation, temperature, and the availability of hand-washing facilities ${ }^{18}$ Excessively loud noises and bright lights can be stressful to the high-risk neonate.

The role of the occupational therapist in the NICU cannot be understated. Combining the vulnerable infant, distressed parents and technically advanced environment can make the NICU a challenging area to work within. Vergara and colleagues ${ }^{10}$ divided the roles of an occupational therapist into three areas within the NICU context, namely 'infant-orientated', 'family-orientated' and 'therapist-orientated'. Within the area of 'infant-orientated', the occupational therapist would perform duties such as splinting, positioning and modulating the external environment. The area of 'family-orientated' would focus on assisting the parents in effectively fulfilling their parental role, and 'therapist-orientated' refers to the occupational therapist using their time and resources as well as their therapeutic use of self to produce favourable outcomes and prevent burnout ${ }^{10}$. There have been rapid advances in neonatology; therefore, intervention has also quickly evolved; moving from sensory supplementation programmes to a more hands-off approach that prioritised family collaboration ${ }^{12}$. An unmatched case-control, retrospective study that investigated the effect of cultural practices on neonatal survival in Indonesia, found that many cultural factors impacted on the neonate such as inapt antenatal care, specific breastfeeding practices, knowledge of hypothermia and the use of Kangaroo Mother Care (KMC) as well as poor neonatal follow-ups ${ }^{17}$. This study highlighted the importance of family and community education to increase safety for neonates. Services also include discharge planning, neonatal assessments, and education, while the occupational therapist aims to match the infant's capabilities with the surrounding environment ${ }^{19}$. The occupational 
therapist works within an MDT; therefore, roles may overlap with other professions ${ }^{5}$.

In the context of South Africa, there is limited literature on the role and scope of occupational therapy within the NICU setting. A quantitative, cross-sectional, descriptive survey, which focused on the training and role of occupational therapists in NICU, was used to access occupational therapists practicing within South Africa. They were members of the Occupational Therapy Association of South Africa (OTASA) and previous experience in the NICU was not an inclusion criterion ${ }^{7}$. The results indicated that there was a lack of skills and knowledge about the NICU which could be the result of a lack of undergraduate training in this field and/ or uncertainty on the exact role of the occupational therapist in the $\mathrm{NICU}^{7}$. In addition, there is the need for in-depth orientation programmes for new graduates, especially when working in remote rural healthcare settings and specialised environments, which is not always provided to community service profession$\mathrm{als}^{20}$. These highlight some of the underlying causes behind the challenges that community service occupational therapists face in delivering a NICU service.

Additionally, poor awareness and understanding of occupational therapy, as well as overlapping roles with other members of the MDT, negatively affected occupational identity 9 . Occupational identity refers to developing a sense of self and who the individual wants to become through repetitive occupational participation ${ }^{21}$. This is closely related to occupational competence ${ }^{21}$. There is limited literature on how working in the NICU impacts on the CSOT's occupational identity as no previous studies, specifically done in a South African context, is currently available and a need was thus identified to investigate novice occupational therapists' experiences of working in NICUs.

\section{METHODS}

This study's methodology is reported against the relevant domains of the consolidated criteria for reporting qualitative research (COREQ), which is advised for explicit and comprehensive reporting of qualitative studies ${ }^{22}$.

\section{Reflexivity and Research Team}

The first author was a newly qualified occupational therapist, having a year of experience post community service at the time of this study. The study was developed out of her own experiences as a CSOT. Several of the participants were known to the first author in her professional capacity; hence her positionality was declared by a series of reflexive statements through this study. A hybrid stance as researcher-therapist was occupied in this study. Strategies to ensure trustworthiness in this study included reflexive journaling, peer debriefing, investigator triangulation in data analysis and respondent validation, and developing and maintaining an audit trail ${ }^{23}$.

\section{Study Design}

Methodological and Theoretical Orientation: This study followed an explorative qualitative design with the use of in-depth interviews. Three theories formed the basis of the conceptual framework that also aided in the analytical process for this study. This framework was developed using three main theories, namely the Model of Human Occupation ${ }^{24}$, the Theory of Occupational Adaptation $^{25,26}$ and The Ecology of Human Performance ${ }^{27}$. The conceptual framework highlights how the person is inextricably linked to their environment, which places various demands on them. To be competent, the process of occupational adaptation is undertaken to overcome the occupational challenge.

Setting: The study was located in KZN Province of South Africa. Interviews were conducted face-to-face, either at a mutually agreed venue in person or via Skype; with the presence of the first author and participant/s only (there were no non-participants present).

Participants: Homogenous purposeful sampling ${ }^{23}$ was employed to recruit participants who (i) were currently registered with the HPCSA, (ii) had completed their community service at a KZN facility between 2014 and 20I7, and (iii) had a minimum of one month's exposure to a NICU as a CSOT. Of the 12 participants in this study, one was male, and the rest were female $(n=I I)$. The mean age of the participants was 26 years old. Majority of the participants attended the University of KwaZulu-Natal for their undergraduate training $(n=9)$, while others were from Stellenbosch University $(n=I)$, the University of Pretoria $(n=I)$ and the University of Witwatersrand $(n=I)$. Majority of the participants completed their community service at district hospitals $(n=8)$, with a few at regional hospitals $(n=3)$ and one at a tertiary hospital. Most participants had a year of NICU exposure $(n=8)$ while the others had between three to five months. The participants completed their community service across nine different districts in $\mathrm{KZN}$, with more than half $(n=7)$ participants reporting that they had permanent supervision by an occupational therapist. Few participants $(n=3)$ had attended additional formal training on neonatal intensive care during their community service year.

Data Collection: A biographical questionnaire was completed prior to the interviews. In-depth interviews followed. These included individual Skype interviews $(n=3)$, individual face-to-face interviews $(n=4)$, one dyad and one triad interview. The afore-mentioned conceptual framework (Figure I) informed the interview schedule; with questions centred round the person (volition, habituation, and performance capacity), environment (physical and social) as well as outcomes (competence, identity and adaptation) related to NICU practice as a CSOT. The interviews spanned a maximum of 90 minutes and were audio-recorded, and manually transcribed.

\section{Data analysis}

Data were analysed thematically ${ }^{23,28}$ by inductive reasoning initially, resulting in first level codes followed by categories identified by the first author. These were exposed to review and critique by two additional coders (co-authors) as part of investigator triangulation. These were then subjected to categorisation via deductive reasoning using the theory of occupational adaptation ${ }^{25}$ and aligned to the conceptual framework (Figure I, p30) by all coders. Respondent validation occurred by ensuring that participants had the opportunity to view the resultant themes.

\section{Ethical considerations}

Ethical clearance was obtained from a Biomedical Research Ethics Committee (BE084/18), and the Health Research and Knowledge Management directorate of the KZN Department of Health (DOH) (KZ_201804_003). Ethical principles adhered to in the study included confidentiality (by the use of pseudonyms and limited linking and de-identified data); autonomy (through voluntary participation and informed consent), and ensuring the participants were aware of their right to withdraw without prejudice.

\section{Trustworthiness}

Trustworthiness was ensured by using purposeful sampling ${ }^{23}$ techniques to enrich the content of the data collected and to heighten credibility. A secure setting was created during the 


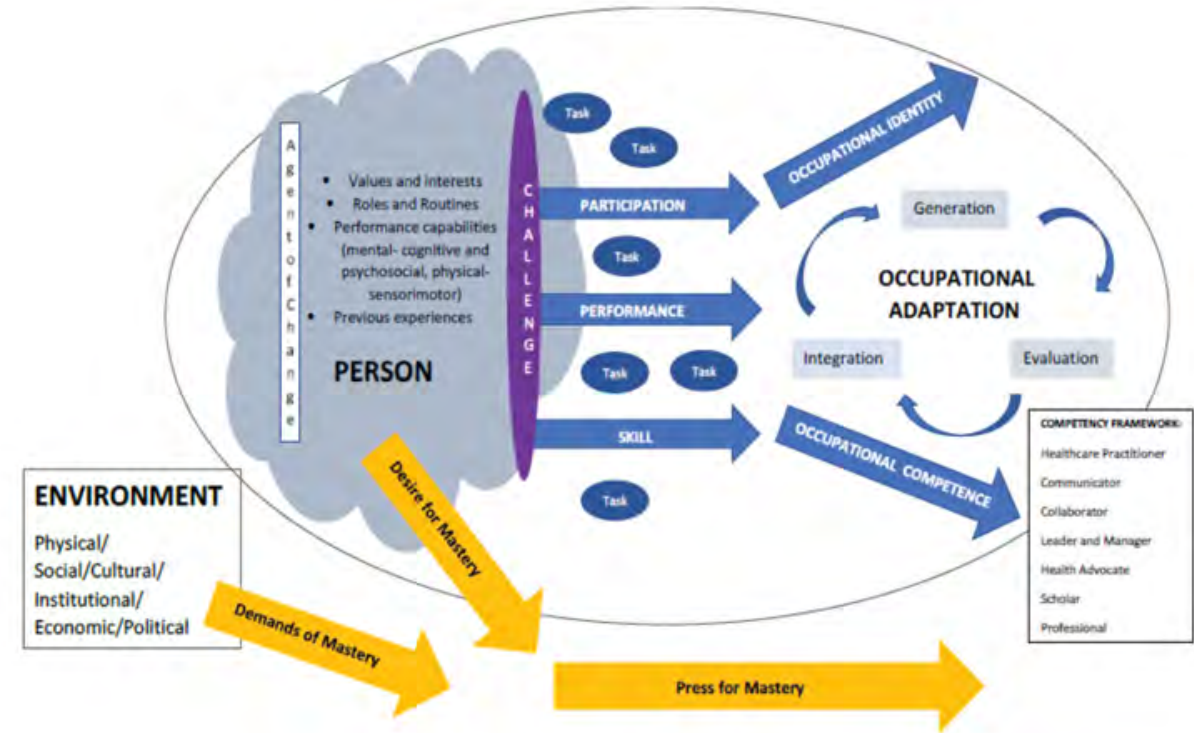

Figure I Conceptual Framework informed by three theories ${ }^{24-27}$

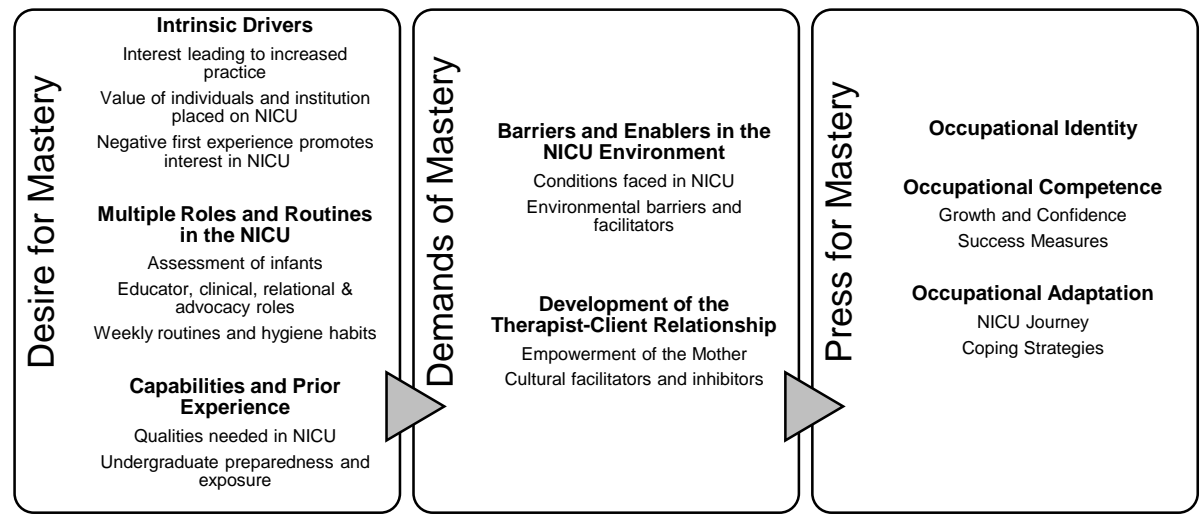

Figure 2 Overview of Themes and Sub-categories that emerged from the data analysis

interviews to increase the likelihood of honest and genuine responses. The interviews were audio-recorded on a digital recorder and transcribed allowing for the use of verbatim quotes. The same interview schedule, guided by the conceptual framework (Figure labove) was used with all participants, with additional probing questions to facilitate deeper discussion. This allows for the research to be replicated to ensure dependability. Sampling continued until redundancy was reached. Confirmability was ensured through reflexivity (via reflexive statements and journaling) and analyst triangulation ${ }^{23}$ (all three authors assisted in the data analysis and interpretation). Transferability was ensured through the use of thick description of the methods, findings, and data analysis as part of the audit trail ${ }^{23}$.

\section{Findings and Discussion}

The study findings resulted in three emergent themes and related sub-categories (Figure 2).

These are augmented by relevant verbatim quotes [Table I, p3I] and supported by the available empirical literature. Generally, newly qualified CSOTs have to overcome the challenges of working in the highly technical environment of the NICU, despite their lack of advanced knowledge, skills and prior experience. The therapists revealed progression from the experience of challenges in fulfilling roles in the NICU, towards a desire to master the environment and gaining more confidence in the process. The working environment created pressures and demands for them to deliver services, which inevitably led to the adoption of coping strategies, thereby enabling adaptation to their roles as occupational therapists in NICUs.

\section{Desire for Mastery}

Every professional, especially when newly qualified, has the innate desire for mastery and competence in their chosen field of work ${ }^{29}$. A perceived occupational challenge acts as motivation to generate an adaptive response to become competent ${ }^{30}$. The participants in this study experienced a negative first visit to the NICU due to feelings of incompetence. Schultz ${ }^{31}$ highlights this as part of the evaluation phase in the internal adaptive response. Intrinsic factors also influenced the CSOTs' desire to excel in the tasks in their environment. The passion and volition of the CSOTs, which is described as the driving force behind initiating a $\operatorname{task}^{30,32}$, influenced their level of involvement in the NICU and their process of occupational adaptation. Those who were interested in paediatrics sought to develop their competence in this area. This was achieved by becoming autonomous learners, seeking mentorship from more experienced professionals, and attending additional courses or in-service training. Additionally, the participants had to be resilient and emotionally strong and had to be able to communicate openly with team members. Other participants mentioned the need to be empathetic and supportive of the parents. These findings corroborate Eraut's ${ }^{30}$ finding that learning from others is the most common means of on-the-job training.

The participants reported a few enabling factors of the physical environment, including the warmth, cleanliness, and suitable hygiene protocols. Some participants found it beneficial to have the mothers staying close to the NICU so that they were able to get more involved with their infants and increase the mothers' sense of empowerment. Despite many reporting being at underresourced facilities, the majority stated that they could always use whatever they had available to carry out their intervention. Some participants did have KMC wraps and proper nesting equipment, whereas others used linen and towels.

The need to fulfil pre-determined professional roles was another intrinsic factor identified. Assessment to identify potential developmental delays and abnormalities was an important occupational therapy role, which concurs with findings in an earlier South African study ${ }^{7}$. According to Vergara et al. ${ }^{10}$, the NICU occupational therapist should be competent in both formal and informal assessments of neonates. Despite many formal assessment tools being used internationally in NICUs ${ }^{12}$, CSOTs mainly used informal observations. This could impact the comprehensiveness and reliability of assessments, as standardised 
Passion for paediatrics and NICU-related practice increased CSOTs drive to learn, gain knowledge and develop networks within this environment. "I've always loved babies and I think it kind of comes easy ...um, when you do have quite a passion for babies" (Nosipho). Interest increased through repeated exposure, positive role models and attending further training. "I wanted to know more ...um, and I wanted to be, to have more skills and more, more knowledge myself to give to moms, to nurses... so I think the interest in it drove me to want to know more" (Emma).

CSOTs placed high value of working in the NICU due to realising the vulnerability of the infants and benefits of early childhood intervention. "You can really make a difference in those early times, with early intervention" (Emma). As well as understanding how the initial NICU contact improved follow-up adherence. "For them knowing that they have our support and that we have the baby and their best interests at heart really helped with follow-ups after they got discharged" (Belinda).

First encounters in the NICU as CSOTs were negative due to lack of knowledge, skills, and limited prior exposure to a NICU. "Yeah, very overwhelmed. Lots of beeping, lots of very tiny babies, I'd never seen babies that size in my life" (Busisiwe). "I felt very out of place, I didn't feel like I necessarily had a role there" (Derryn). "I felt useless (laughs)... I was so scared I was going to break the baby" (Sanaah).

\section{MULTIPLE ROLES AND ROUTINES IN THE NICU}

CSOTs emphasised assessment of infants, using informal observations in the NICU (infant's reflexes, muscle tone, sleep states, stress cues, abnormal movements, vital signs, and range of motion). "My role in the NICU it was more of a [n] assessment, less treatment" (Aphiwe). Formal assessments included the WITS Developmental profile and the Neonatal Movement Assessment. Use of standardised protocols in the NICU was limited, "I didn't know of any specific assessments and I didn't know of any specific protocols except the kangaroo care" (Michelle) and some participants used departmental screening tools and protocols, "In our department there was no protocol to work with so I had to develop my own protocol" (Aphiwe).

Roles included engagement with parents, caregivers, family members, NICU staff and fellow occupational therapists. "I also think we have $a$ role in educating nurses and doctors on structural and environmental factors ... like we're the only ones who will come in and make that sort of change" (Derryn). Educational content focused on handling and positioning of the infant, swaddling, developmental milestones, environmental considerations, mother-infant bonding, stress cues and self-soothing as well as kangaroo mother care. "But we wouldn't go in and do anything if the mom wasn't there cause we were educating her the whole time" (Lydia).

Clinical roles in NICU included positioning, containment hold, passive stretching, nesting, sensory stimulation, baby massage and splinting and developmental follow-up post-discharge. "I would position the babies, so often you would find them ...um, splayed out like little spatchcock chickens or butterflies ...um, and I would just go turn them and put them into a more side-lying position" (Michelle). "I do think there is a role for us to provide developmental follow-up, I think that's a really important role" (Derryn).

The relational role of therapists included support of mothers in their parental role through confidence-building and acting as mediator between other NICU staff and parents. "I think that was basically our main role is just to make the mom feel empowered to know what's wrong with her baby" (Belinda). As well as advocacy for changes to the environment and agency for the occupational therapists' role in NICUs. "I spent the first two months just trying to advocate for OT services in the NICU, so I found it very, very intimidating and very challenging" (Reeya).

Routines and habits established within the NICU included scheduling daily visits during feeding times, documenting progress in files, dialogue with staff members, ward rounds and observing infection control protocols. "So, there's a lot of hygiene restrictions...so you had to put on all the aprons and the gloves and the whatnot, wash all your hands before you go in" (Derryn).

\section{CAPABILITIES AND PRIOR EXPERIENCE}

The need for confidence, good time management skills, empathy, emotional resilience, creative problem solving and willingness to learn, were emphasised by CSOTs when working in the NICU. "You needed to be very understanding, you need empathy ... um, you need to be confident because the babies can feel and the moms can see if you confident or not" (Michelle). "You definitely have to be resilient. Because not all these children make it, which was very tough for me in the beginning" (Emma). CSOTs felt underprepared for work in the NICU due to limited or no knowledge or skills from an undergraduate training level. "I think we had a very good lecturer ... but it was the actual practicality of the hands-on ... that I feel was definitely lacking” (Reeya).

\section{BARRIERS AND ENABLERS IN THE NICU ENVIRONMENT}

CSOTs indicated that the physical environment was conducive for work and they were able to adapt to under-resourced departments. "It didn't have a lot of resources. We used what we had, we used what we could. I also bought in a lot of my own stuff from home that I had acquired over the years of studying" (Emma). Having mothers accommodated close to the NICU allowed for facilitation of mother-infant bonding and in enhancing the mother's sense of empowerment. The NICU was reported as 'over-stimulating' (loud noises and bright lights), having limited space, uncomfortable seating and a depressing atmosphere. High patient loads, lack of privacy, and having to plan intervention around ward schedules were some additional challenges experienced. "The tin bins opening and closing all the time and the nurses speaking loudly, it wasn't very conducive I wouldn't say it was a mother-baby friendly environment” (Pearl).

\section{DEVELOPMENT OF THE THERAPIST-CLIENT RELATIONSHIP}

CSOTs reported therapeutic rapport building with mothers as essential in the carry-over of intervention, follow-up and long-term adherence. "You're going in, building relationship with the mom ...um, and you know that's going to have a much better carryover, I mean if, if we just went in there and didn't even talk to the moms, like that really has such a limited impact" (Lydia). Some CSOTs indicated language barriers as affecting educational efforts. Patients hailed from low socio-economic environments and with limited education. "So, a lot of these ladies [mothers] were not highly educated. Some of them couldn't read, so pamphlets don't work and things like that ...um, so drawing pictures and just teaching them every day how to handle, how to work with their little ones" (Nikita). A high rate of teenage pregnancy, poor pre-natal treatment adherence and poor family planning were noted. Mothers with post-natal depression were challenging to work with as they became less responsive. "If some of my moms became depressed after birth ...um, that l, it would be a struggle to get them to do KMC [Kangaroo Mother care]" (Busisiwe). 


\section{OCCUPATIONAL IDENTITY}

CSOTs reported clinical practice in the NICU broadened their understanding of the diversity of the profession and for some, validated their role as occupational therapists within the setting. "I think it gave me pride that we had a role in these little human's lives, from like the real beginning. We could have a positive impact on something" (Busisiwe). It provided a distinct role from other healthcare professions. "I think sometimes with, with occupational therapy, you kind of feel like 'Ah okay, with physical we kind of overlap with a physio sometimes' but it was really cool to have such defined role and a presence in the NICU [neonatal intensive care unit]" (Lydia).

\section{OCCUPATIONAL COMPETENCE}

CSOTs reported initial incompetence for NICU practice, but found that their competence had increased by the end of the year. "Well definitely in the beginning not competent or confident at all. But then like by the end like, like I wouldn't say I felt like completely competent, like I felt I still had so much to learn, but I felt a hundred times better" (Sanaah). Measuring success of intervention in the NICU setting was difficult, due to high turnover rates and poor adherence to intervention. Success was mostly noted by frequency of follow-ups and with comparison to normal developmental milestones over the long-term. "A lot of moms didn't follow-up and come to appointments and so, it's difficult to measure the child's progress when you're not seeing them regularly" (Emma).

\section{OCCUPATIONAL ADAPTATION}

CSOTs underwent a process of adaptation during their exposure within NICUs and reported more efficient planning and independence in practice. "The more time I spent there the more confident I became in my, in my role, what I can offer to-to parents and so it became a lot more efficient (laughs) ...um, and a lot more, I became a lot more confident" (Emma). CSOTs used internet resources, university notes and textbooks for upskilling, in additional to relying on knowledgeable supervisors as resources. "Luckily I had a supervisor who basically showed me the ropes because otherwise I would have no idea what to do" (Nosipho). Additional courses were also described as useful in improving clinical practice. "So, l'd kind of gone on the course in the middle of the year so l'd already been in the neonatal intensive care unit and I could kind of reflect on how it had been in neonatal intensive care and use that, and then going back at the end of the year, just felt so much more equipped to, to be there" (Lydia). Courses attended had to be prioritised based on their caseload, personal interests, and in areas they felt most incompetent. Relevant courses were noted to be limited and expensive. "I also don't remember them like offering any in our area.. you're always waiting for the ones to come to Durban" (Nosipho).

tools guide more holistic, consistent, valid and reliable results.

Other essential roles included clinical and relational/ consultancy roles, which were similar to other studies $^{7,10}$. Clinical roles included positioning and handling neonates, facilitating $\mathrm{KMC}$, providing family support and modifying the environment, aligned with the findings of Müller et al. Many of the CSOTs advocated for a less stimulating environment. The environment is significant as it substitutes for the intra-uterine environment for a pre-term infant and plays a role in shaping the infant's developing central nervous system ${ }^{33}$. It is widely accepted that occupational therapists are involved with environmental adaptations, even in NICUs ${ }^{10}$. The participants mainly focused on auditory and visual adaptations, as constant bright lights and loud noises can be harmful to neonates and cause significant distress ${ }^{33}$. However, limited attention was paid to olfactory, gustatory, proprioceptive-vestibular, and tactile stimuli in this study ${ }^{10}$ which are also important sensory aspects that could impact neonatal stress.

The participants' educational efforts were directed predominantly at mothers or caregivers and towards increasing awareness of the role of occupational therapy with team members. The educational and advocacy roles of CSOTs in a NICU in this study differed from Müller et al. 's ${ }^{7}$ study. The different findings were attributed to new graduate occupational therapists not having sufficient experience to deal with the resistance encountered.

\section{Demands of Mastery}

As the CSOTs moved towards mastery, specific demands were placed on them that were contextually dependent, creating an occupational challenge. Participants reported facing high patient loads and lack of adequate time to fulfil all duties, which negatively influenced service delivery in the NICU. This highlighted the need for on-the-job training and mentorship. The lack of privacy when dealing with mothers was unsuitable to building relationships. Establishing a trust relationship between the mother and the therapist is vital to ensure compliance with interventions. Lincare ${ }^{18}$ stated that counselling rooms and comfortable seating for mothers should be accessible in all units. However, many participants did not experience this at their facilities and recognised it as a necessity.

Family-centred intervention has become the gold standard for the healthcare of infants ${ }^{34}$, with greater parent responsiveness and warmth being correlated with improved developmental outcomes ${ }^{35}$. The occupational therapist's role in the NICU should be both infant- and parent-orientated ${ }^{12}$. The CSOTs aimed at forming strong relationships with the parents and focused on parent education and empowerment. This was especially important in the KZN setting, where the low maternal education levels, poverty, younger age of mothers and a language barrier were noted as hindrances to early childhood development programmes, in keeping with Clements and colleagues study ${ }^{36}$. Most of the participants reported that it was difficult to measure the success of their intervention in the NICU setting. Most of the participants measured their success by the frequency of follow-ups and measuring developmental milestones over the long-term, which proved difficult due to high turnover rates and poor compliance of parents. Despite many CSOTs providing developmental follow-ups at primary healthcare (PHC) clinics with the infant's primary caregiver, there was little other community engagement or education, correlating with another South African study ${ }^{37}$. This could be attributed to a lack of undergraduate training on relevant $\mathrm{PHC}$ services in infant health.

According to the HPCSA ${ }^{38}$, undergraduate training programmes in South Africa must equip health science students to fulfil the roles of a healthcare practitioner, communicator, collaborator, manager, leader and advocate, scholar, and professional. The participants agreed that occupational therapists require more than just knowledge in the field to work effectively in NICUs. In the KZN context, 
particular importance was placed on perseverance and creative problem-solving skills, needed for dealing with under-resourced facilities, a lack of undergraduate training and the lack of awareness of the occupational therapist's role in NICUs. This could be due to the poor understanding of the occupational therapist's role in the NICU within South Africa, as well as the limited policies in place to generate relevant protocols ${ }^{7}$.

\section{Press for Mastery}

The study found a press for mastery throughout the community service year, as the CSOTs developed their identity and competence and sought ways to cope with NICU practice. Initially, the CSOTs felt incompetent and ill-equipped in dealing with neonates. However, through exposure to NICU tasks, identity was built, which shaped the CSOTs' interactions in this environment. Many participants also felt that their work in NICU made them more holistic as therapists and felt they made a difference. A few of the participants believed that delivering services in the NICU increased their pride in the occupational therapy profession as they viewed their role as valuable. Therefore, the CSOTs' identity was built through personal motivators, social support, and feelings of productivity or success ${ }^{21}$. The CSOTs felt an increased sense of identity when there was less overlap with physiotherapists. In contrast, poor understanding of the occupational therapist's role challenged their identity, concurring with Van Stormbroek and Buchanan's study.

Participants reported a lack of orientation to their new environment, which is required to improve feelings of confidence ${ }^{20}$. According to Hodgetts and colleagues ${ }^{29}$, it takes a therapist six months to two years to develop feelings of competence. Increased competence at the end of community service was evidenced when CSOTs needed less supervision, integrated their skills, applied learnt knowledge, and made dynamic changes in the environment, mainly through in-services.

The CSOTs sought methods to adapt to the NICU demands, including research using internet and textbook resources and contacting supervisors or mentors. Internet resources are commonly used to enhance professional development, especially in our technological advancing world $39,40,41,42$. They are beneficial to professionals needing to access information rapidly and cost-effectively, especially in rural areas ${ }^{40}$. This study found Google and YouTube were familiar internet sources used to obtain information, which correlates with previous studies ${ }^{39,41}$. Authenticity and accessibility of the online information are essential to consider as some information may be outdated, not evidence-based, or even irrelevant to the context ${ }^{40}$. This study highlighted the lack of permanent supervision for CSOTs, which correlated with Van Stormbroek and Buchanan's ${ }^{9}$ findings. External mentors are beneficial to CSOTs, who have no supervisors at their facility ${ }^{18}$. This is often the case in South Africa, where there is inadequate social, administrative, and clinical support for CSOTs ${ }^{20}$. Therefore, providing contact lists of experienced occupational therapists familiar with KZN NICUs, who have gained advanced skills in this area of practice, could be a more practical solution in this context.

Very few participants attended NICU-related courses as CSOTs. Nevertheless, those who did, stated this was invaluable for their clinical practice. Low attendance could be attributed to the poor availability of NICU-related courses in KZN, and limited finances. The most common course attended was the 'Little Steps: Neurodevelopmental Supportive Care of the
Preterm Infant', similar to Müller et al.'s ${ }^{7}$ findings. The training recommendations reported in this study included provision of affordable NICU-related courses for CSOTs. Additionally, it was recommended that undergraduates are exposed to NICU indirectly, through practical videos or NICU-related projects, and sensitised to the negative effects of poor clinical practice with neonates.

\section{CONCLUSIONS}

The desire, demand and press for mastery by occupational therapists working in the field of neonates were highlighted in this study. Newly qualified CSOTs appear to be able to overcome the challenges of working in the NICU's highly technical environment, despite their lack of advanced knowledge, skills, and prior experience, by the end of their community service year. In this study, the participants' first negative visit to the NICU and their innate passion for paediatrics, were drivers towards their need to become more competent in the field. Personal characteristics such as emotional resilience, empathy and open communication were essential to work in NICUs. However, high patient loads and lack of adequate time to fulfil all duties negatively influenced service delivery in the NICU and highlighted the need for on-the-job training and mentorship as support. A focus on establishing a strong rapport with the parents and on parent education was noted, which resulted in enhanced compliance with intervention, especially given the young age and low education level of several mothers.

Occupational identity appeared to be established on personal motivators and feelings of productivity. A few of the participants believed that working in the NICU increased their pride in the occupational therapy profession as they felt their role was invaluable. The participants experienced an increased perception of competence by the end of community service. They believed they required less supervision, integrated their skills, applied learnt knowledge, and made active changes in the environment. The coping methods used to increase this sense of competency included conducting further research on unknown topics, consulting supervisors or mentors and attending NICUrelated courses as CSOTs.

\section{LIMITATIONS}

Transferability is limited due to the study being conducted in the province of $K Z N$ with a small sample size. Although the training and experiences of an occupational therapist practicing in $\mathrm{KZN}$ may be applicable in various provinces in South Africa, the health system variances, including availability of resources and infrastructure, may also limit transferability. Findings are limited to the experiences of CSOTs between 2014 and 20I7, with many participants from the same university, which could have biased the results. A response bias may have been created as occupational therapists with a negative attitude towards neonatal intensive care or who felt incompetent in this practice area may not have responded to the invitation to participate.

\section{ROLE OF AUTHORS}

Michaela Hardy was the primary investigator in this study and was responsible for study design, sourcing relevant literature, contacting, and interviewing participants, transcribing interviews, and data analysis. Pragashnie Govender and Deshini Naidoo were supervisors of the study who assisted in the conceptualisation and design of the study, data analysis and interpretation together with Michaela Hardy. All authors contributed to the drafting of the manuscript. 


\section{REFERENCES}

I. World Health Organisation [WHO]. Nurturing care for early childhood development, A framework for helping children survive and thrive to transform health and human potential. 2018.

http://www.who.int/maternal_child_adolescent/documents/ nurturing-care-early-childhood-development/en/ (16 September 20I8).

2. Hoque M, Haaq S, Islam, R. Causes of neonatal admissions and deaths at a rural hospital in KwaZulu-Natal, South Africa. The South African Journal of Epidemiology and Infection. 201 I: 26(I), 26-29. https://www.tandfonline.com/doi/abs/10.1080/10158782.20I I.II 441416 ( 15 July 2017).

3. Department of Health, Republic of South Africa. Maternal and Child Health. 2017. http://www.health.gov.za/index.php/gf-tbprogram/I I3-maternal-and-child-health (7 August 20I7).

4. Askin DF, Wilson D. In Wong's Nursing care of infants and children. Chapter 10, The High-Risk New-born and Family. 20I I: 314-389. https://coursewareobjects.elsevier.com/objects/evolve/E2/book_pages/wongncic/docs/Hockenberry_Chapter I0.pdf ( 15 July 2017).

5. Barbosa BM. Teamwork in the Neonatal Intensive Care Units. Physical and Occupational Therapy in Paediatrics. 2013: 33(I): 5- 26. http://www.rheapaul.com/Files/Teamwork\%20in\%20NICU.pdf (27 July 2017).

6. Hunter J, Lee A, Altimier L. Chapter 2I, Neonatal Intensive Care Units. In Case-Smith J, O'Brien, JC. Occupational Therapy for Children and Adolescents. Seventh Edition. 20I5: 595- 635.

7. Müller M, Myburgh A, Stock R. The Training and Role of Occupational Therapists in South African Neonatal Intensive Care Units. Unpublished manuscript. Submitted to the Faculty of Health Sciences, School of Therapeutic Sciences, University of the Witwatersrand, Johannesburg, in partial fulfilment of the Requirements for the Bachelor of Science (Occupational Therapy). 2016.

8. Dewire A, White D, Kanny E, Glass R. Education and Training of Occupational Therapists for Neonatal Intensive Care Units. American Journal of Occupational Therapy. 1996: 50(7): 486- 494. https://www.ncbi.nlm.nih.gov/pubmed/881 9600 (27 July 2017).

9. Van Stormbroek K, Buchanan H. Community Service Occupational Therapists: striving or just surviving?' South African Journal of Occupational Therapy. 2016: 46(3): 63- 72.

http://www.scielo.org.za/scielo.php?script $=$ sci_ arttext\&pid $=$ S23 I 0-383320 I $60003000 \mathrm{I}$ I \&lng = pt\&nrm $=$ iso $(28$ July 2017).

10. Vergara E, Anzalone M, Bigsby R, Gorga D, Holloway E, Hunter J. Specialized Knowledge and Skills for Occupational Therapy Practice in the Neonatal Intensive Care Unit. American Journal of Occupational Therapy. 2006: 60, 659-668. http://ajot.aota.org/article.aspx?articleid = I8700 I I (I 5 July 2017).

II. Gauchan E, Basnet S, Koirala D P, Rao KS. Clinical profile and outcome of babies admitted to Neonatal Intensive Care Unit (NICU). Journal of Institute of Medicine. 20I I: 33(2). http://jiom.com.np/index.php/jiomjournal/search/authors/view?firstName=Gauchan\%20E\%2C\%20Basnet\%20S\&middleName = \&lastName $=$ Koirala\%20D\%20P\%2C\%20 Rao\%20K\%20S\&affiliation=\&country= (I5 July 20I7).

12. Gorga D. The Evolution of Occupational Therapy practice for infants in the Neonatal Intensive Care Unit. The American Journal of Occupational Therapy. 1994: 48 (6), 487-489.

https://ajot.aota.org/article.aspx?articleid = 1873289 (23 January 20I8).

13. Lloyd L, De Witt W. Neonatal mortality in South Africa: How are we doing and can we do better? Editorial. South African Medical Journal. 2013: 103(8), 518-519.
http://www.samj.org.za/index.php/samj/article/view/7200/528I (I I February 2019).

14. South African Speech-Language- Hearing Association [SASLHA]. Guidelines: Early Communication Intervention. Ethics and Standards Committee. 20II.

https://www.mm3admin.co.za/documents/docmanager/55e836d53332-4452-bb05-9f। 2be8da9d8/000 I 2503.pdf (I 5 July 20 I7).

15. Province of KwaZulu-Natal. Socio-economic Review and Outlook 2015/2016. 2016. http://www.kzntreasury.gov.za/Socio\%20Economic/SERO_FINAL_4_March_2016.pdf (2 August 2017).

16. Dayal H. Provision of rehabilitation services within the District Health System-the experience of rehabilitation managers in facilitating this right for people with disabilities. South African Journal of Occupational Therapy. 2010: 40(I), 22-26.

17. Sutan R, Berkat $S$. Does cultural practice affect neonatal survival- a case control study among low birth weight babies in Aceh Province, Indonesia. BMC Pregnancy and Childbirth. 2014: I4(342).

https://www.ncbi.nlm.nih.gov/pubmed/25269390 (I 2 August 2018).

18. Lincare. Norms and Standards for Essential Neonatal Care. 2013. http://www.lincare.co.za/wp-content/uploads/2016/06/Chapter2-Norms-and-Standards-for-Essential-Newborn-Care.pdf (7 August 2017).

19. Nightlinger K. Developmentally Supportive Care in the Neonatal Intensive Care Unit: An Occupational Therapist's Role. Neonatal Network. 201 I: 30(4), 243-248.

https://search.proquest.com/openview/f9d328c0 I d69f3 I c5fb I 230 $6 \mathrm{de} 53 \mathrm{I}$ 50a/ $/$ ?pq-origsite $=$ gscholar \&cbl $=646499$

(II February 2019).

20. Beyers B. Experiences of community service practitioners who are deployed at a Rural health facility in the Western Cape. Unpublished manuscript. A mini thesis submitted for the degree of Magister Curations at the School of Nursing, Faculty of Community and Health Sciences, University of the Western Cape. 2013. http://etd.uwc.ac.za/xmlui/bitstream/handle/ I I 394/332 I/Beyers_MCUR_20I3.pdf?sequence $=$ I (28 July 2017).

21. Phelan SK, Kinsella EA. Occupational identity: Engaging sociocultural perspectives. Journal of Occupational Science. 2009: I6(2), 85-9I. file:///D:/Users/micst/Downloads/PhelanKinsella2009JOS.pdf (12 August 2018).

22. Tong A, Sainsbury P, Craig J. Consolidated criteria for reporting qualitative research (COREQ): a 32-item checklist for interviews and focus groups. International journal for quality in health care. 2007 Dec I; I9(6):349-57.

23. Patton MQ. Qualitative research \& evaluation methods: Integrating theory and practice. Sage publications; 2014 Oct 29.

24. Kielhofner G. A model of human occupation: Theory and application. Lippincott Williams \& Wilkins; 2002.

25. Schkade JK, Schultz S. Occupational adaptation: Toward a holistic approach for contemporary practice, part I. American Journal of Occupational Therapy. 1992 Sep 1;46(9):829-37.

26. Schultz S, Schkade JK. Occupational adaptation: Toward a holistic approach for contemporary practice, part 2. American Journal of Occupational Therapy. 1992 Oct 1;46(10):917-25.

27. Dunn W, Brown C, McGuigan A. The ecology of human performance: A framework for considering the effect of context. American Journal of Occupational Therapy. 1994 Jul 1;48(7):595-607.

28. Clarke V, Braun V, Hayfield N. Thematic analysis. Qualitative psychology: A practical guide to research methods. 2015 Jan I:222-48.

29. Hodgetts S, Hollis V, Triska O, Dennis S, Madill H, Taylor E. Occupational therapy students' and graduates' satisfaction with professional education and preparedness for practice. The Canadian Journal of Occupational 
Therapy. 2007.

https://www.researchgate.net/profile/Sandra_Hodgetts/publication/6221920_Occupational_Therapy_Students\%27_and_Graduates\%27_Satisfaction_with_Professional_Education_and_Preparedness_for_Practice/links/5877a3b94585 I58d8a6c I 7d5/OccupationalTherapy-Students-and-Graduates-satisfaction-with-Professional-Education-and-Preparedness-for-Practice.pdf?origin=publication_detail (19 October 2018).

30. Eraut M. How Professionals Learn through Work. University of Surrey. 2008. http://surreyprofessionaltraining.pbworks.com/f/How +Professionals+Learn+through+Work.pdf (I November 2018).

31. Schultz S. Theory of Occupational Adaptation. In Crepeau EB, Cohn ES, Schell BA. Willard's \& Spackman's Occupational Therapy. I I th Edition. Philadelphia. Lippincott Williams \& Wilkins. 2009.

32. Forsyth K, Kielhofner G. Model of Human Occupation. Ergoterapeuten. 2008.

https://www.noexperiencenecesarybook.com/oQEb2/the-articlepresents-a-current-overview-of-the-ergoterapeuten.html (I2 October 2017).

33. Aucott S, Donohue PK, Atkins E, Allen MC. Neurodevelopmental care in the NICU. MRDD Research Reviews. Mental Retardation and Developmental Disabilities. 2002.

https://pdfs.semanticscholar.org/b04e/579976367540682cc58023 Of3075244784db.pdf (7 February 2018).

34. King G, Strachan D, Tucker M, Duwyn B, Desserud S, Shillington M. The Application of a Transdisciplinary Model for Early Intervention Services. Infants and Children. Wolters Kluwer Health. Lippincott Williams \& Wilkins, 2009.

https://depts.washington.edu/isei/iyc/22.3_King.pdf (4 November 20I8).

35. Hadders-Algra M. Challenges and limitations in early intervention. Developmental medicine and child neurology. Netherlands, 201 I. http://onlinelibrary.wiley.com/doi/10.1 I I I/j.14698749.201 I.04064.x/pdf (I5 July 2017).

36. Clements KM, Barfield WD, Kotelchuck M, Wilber M. Maternal SocioEconomic and Race/Ethnic Characteristics Associated with Early Intervention Participation. Maternal Child Health Journal. 2008.

https://www.ncbi.nlm.nih.gov/pubmed/I8026825 (29 September 2018).

37. Naidoo D, Van Wyk J, Joubert RW. Exploring the occupational therapist's role in primary health care: Listening to voices of stakeholders. African Journal of Primary Health Care Family Medicine. 2016: 8(I). http://www.scielo.org.za/pdf/phcfm/v8nl/30.pdf (4 November 2018).

38. Health Professions Council of South Africa [HPCSA]. Core competencies for undergraduate students in clinical associate, dentistry and medical teaching and learning programmes in South Africa. 2014. http://www.hpcsa.co.za/uploads/editor/UserFiles/downloads/ medical_dental/MDB\%20Core\%20Competencies\%20-\%20 ENGLISH\%20-\%20FINAL\%2020I4.pdf (29 September 2018).

39. MacWalter G, McKay J, Bowie P. Utilisation of internet resources for continuing professional development: a cross-sectional survey of general practitioners in Scotland. BMC Medical Education. 2016. https://www.ncbi.nlm.nih.gov/pmc/articles/PMC4721 I89/ pdf/ I2909_2016_Article_540.pdf (2I October 2018).

40. Herrington AJ, Herrington JA. Using the internet for professional development: the experience of rural and remote professionals. University of Wollongong. Australia. 2006.

https://ro.uow.edu.au/cgi/viewcontent.cgi?referer = https://www.google.co.za/\&httpsredir $=\mid$ \& \&article $=|9| \mid$ \&context =edupapers (2I October 20I8).

4I. Govender P, Mostert K. Making sense of knowing: Knowledge cre- ation and translation in student occupational therapy practitioners. African Journal of Health Professions Education. 20 I 9; I I (2):38-40.

42. Naidoo D, Govender P, Stead M, Mohangi U, Zulu F, Mbele M. Occupational therapy students' use of social media for professional practice. African Journal of Health Professions Education. 2018; I0(2):101-5.

Corresponding Author

Michaela Hardy

Email: michardy22@gmail.com 